\title{
Collecte des langues des signes des sourds de Soure (île de Marajó): un parcours méthodologique (2008/2013), les enjeux sociaux et politiques de la non reconnaissance des langues des signes émergentes pratiquées par ces sourds
}

\author{
Coleta de línguas de sinais dos surdos de Soure (Ilha de \\ Marajó): um percurso metodológico (2008/2013), os desafios \\ sociais e políticos do não-reconhecimento das línguas de sinais \\ emergentes destes surdos
}

\author{
Maria Luizete Sampaio Sobral CARLIEZ \\ École d'Application de l'Université Fédérale de Pará (EAUFPA) \\ Ivani FUSELLIER \\ Université Paris-VIII
}

\begin{abstract}
RÉSUMÉ: Le présent article est le résultat d'une expérience de collecte des langues des signes (LS) pratiquées par les sourds de Soure/Île de Marajó-PA, entre les années de 2008 et 2013, selon l'approche linguistique des LS de l'Université Vincennes Saint Denis - Paris 8, où j'ai fait un stage postdoctoral sur la méthodologie de la collecte en LS. Dans une première étape, nous faisons un aperçu historique dont le parcours illustre la motivation sur notre choix thématique et comment nos observations empiriques ont évolués vers un travail plus structuré. Cette recherche vise ainsi exposer les acheminements méthodologiques de la collecte des LS des sourds de Soure, d'après un regard social et politique de cette communication gestuelle et visuelle intrinsèquement ancrée dans leur culture.
\end{abstract}

MOTS CLÉ : Langues des signes émergentes. Méthodologie. Collecte. Culture.

RESUMO: O presente artigo é o resultado de uma experiência de coleta de línguas de sinais (LS) praticadas pelos surdos de Soure/Ilha do Marajó-PA, entre os anos de 2008 e 2013, segundo a abordagem linguística das LS da Universidade Vincennes Saint Denis - Paris 8, onde cursei um estágio pós-doutoral sobre metodologia da coleta em LS. Em uma primeira etapa, fazemos uma percepção histórica cujo percurso ilustra a motivação de nossa escolha temática e como nossas observações empíricas evoluíram para um trabalho mais estruturado. Essa pesquisa visa então expor os direcionamentos metodológicos da coleta das LS dos surdos de Soure, sob um olhar social e político dessa comunicação gestual e visual intrinsicamente enraizada em sua cultura.

PALAVRAS-CHAVE: Línguas de sinais emergentes. Metodologia. Coleta. Cultura.

\section{Introduction}

En 2006, lors de la présentation du mémoire de Thianny Brito, une étudiante de l'Université Fédérale de Pará (UFPA), attachée au Campus Universitaire de Marajó/Soure, capitale de l'Île de Marajó, sur l'enseignement du portugais aux sourds 
dans les écoles publiques de cette ville, nous avons constaté que ces personnes n'étaient pas scolarisées de façon adéquate, voire même sans aucune scolarité. Thianny Brito avait donc repéré une trentaine de sourds vivant, pratiquement tous, dans une totale condition d'isolement de communication avec la société locale. Je dirigeais ce campus de l'UFPA depuis 2004 et je n'avais jamais pris connaissance de ces personnes et moins encore de leur cursus scolaire. Nous ne les voyions pas dans les rues de la ville non plus, dans les plages, dans les écoles ou dans les manifestations culturelles de la ville. C'étaient des inconnus pour nous et le rôle des universités fédérales placées dans l'intérieur du Brésil est aussi, au-delà d'offrir une formation supérieure de qualité à la population environnante, celui de favoriser, en partenariat avec le pouvoir municipal local, la mise en place des stratégies de développement social en ce qui concerne surtout l'éducation et la culture locales où l'université est installée, cette stratégie politique éducationnelle rentre dans le cadre des «activités extensionistes » ${ }^{1}$, c'est-à-dire, des activités qui sont développées en tant que projets pour articuler les actions universitaires avec celles du municipe concerné.

Ainsi, en tant que dirigeante de ce campus, j'ai organisé, dans cette même année, un travail d'équipe avec les étudiants universitaires stagiaires, fonctionnaires, professeurs et personnes bénévoles, de la communauté locale pour aller à la recherche de ces sourds. Nous en avons trouvé une cinquantaine dont seulement quatre étaient régulièrement inscrit à l'école Dom Alonso sous la responsabilité du Professeur Marta Bezerra, la seule personne s'occupant de tous les enfants en situation de handicap, qui étaient d'ailleurs mis tous dans une même salle de cours, avec les entendants, sans que l'on se soucie de leurs besoins spécifiques concernant leur apprentissage. En 2003, j'avais fondé l'Association d'Aide à l'Insertion Sociale de Marajó (AMIS MARAJO) dans le but de développer des activités éducationnelles avec les enfants des alentours de l'UFPA et, par conséquent, approcher ces familles de notre environnement académique. Lorsque nous avons découvert ces sourds isolés nous les avons invités à l'association. Notre première idée était de faire en sorte qu'ils se rencontrent entre eux et cela a eu lieu de façon très naturelle, dès qu'ils se sont vus pour la première fois, la gestualité de leurs mains a pris toute la place de leur interaction communicative. Nous ne comprenions rien de ce qu'ils disaient entre eux et nous ne savions guère par où

\footnotetext{
${ }^{1}$ Activités académiques réalisées sur le terrain dans des espaces à l'extérieur de l'université.
} 
commencer. Thianny Brito, qui maîtrisait la LIBRAS ${ }^{2}$, a commencé à leur donner cours de portugais écrit dans l'association et cela a continué jusqu'en 2007 car elle a dû partir de Soure pour travailler dans un autre municipe du Marajó. Tout était très intuitif et sans aucun fondement théorique ou supports pédagogiques.

\section{Parcours historique}

En 2007, j'ai commencé à organiser un Forum International pour discuter les politiques publiques d'insertion des personnes sourdes et aveugles du Marajó au sein de l'UFPA/Campus de Soure. Je suis partie pour la France où j'ai pris connaissance de l'Institut des Jeunes Sourds de Paris (INJS de Paris) et ai ainsi pris contact avec son directeur, Monsieur Jean-François Dutheil qui, par une coïncidence inattendue, avait été invité pour le cent- cinquantenaire de l'Institut National de l'Education des Sourds de Rio de Janeiro (INES), au Brésil. Il s'y rendrait donc en septembre de cette même année. L'INES a été fondé en 1857 par E. Huet, un professeur sourd provenant de France. J'ai profité de cette opportunité pour l'inviter à notre Forum qui aurait lieu au même mois ${ }^{3}$. Il a participé de notre événement à Soure et nous avons commencé à discuter sur la possibilité de signer un partenariat entre l'INJS et l'UFPA. Depuis 2006, lorsque nous avons découvert la réalité des sourds de Soure jusqu'en 2011, nous avons réalisé quatre éditions de ce Forum International pour discuter les politiques publiques pour l'insertion des sourds dans la société locale et dans l'Etat de Pará.

En 2008, j'ai fait un stage d'un mois à l'INJS. En 2008, nous avons envoyé Eder Barbosa, qui était à l'époque notre étudiant de Lettres - Langue Française, pour un stage à l'INJS aussi. Dans cette même année, l'UFPA signe son premier accord de collaboration avec l'Institut National des Jeunes Sourds de Paris, l'Institut National d'Educations des Sourds de Rio de Janeiro et l'Association AMIS MARAJO. Et c'est encore en 2009 que l'INES envoie à Soure douze professionnels pour former les professeurs du réseau public d'enseignement municipal dans le domaine de l'éducation des sourds.

\footnotetext{
${ }^{2}$ Langue des Signes Brésilienne (Língua Brasileira de Sinais).

${ }^{3}$ Ce Forum a eu lieu en septembre 2007 : «Forum International de discussion des politiques publiques pour l'insertion des personnes aveugles et sourds de Marajó ». Seulement ce premier Forum a discuté sur l'insertion des personnes aveugles. Les autres rencontres que nous avons réalisées par la suite se sont restreintes aux personnes sourdes compte tenu de l'ampleur qu'a prise l'événement et de l'inexistence de cas d'aveugle isolé à Soure. Voir images et informations complémentaires dans les annexes.
} 
En juin 2009, l'UFPA/Soure embauche sa première professeur de Langue des Signes Brésilienne, sourde, Ellen Formigosa, et dans cette même année un professeur de Psychologie de l'Education, aussi attaché au projet de création de la licence en LIBRAS et Portugais L2, suit un stage à l'INJS de Paris. En 2010, la professeure de LIBRAS fait, à son tour, un stage dans ce même institut spécialisé à Paris. C'est l'année de la création de la licence susdite et nous avons eu 40 étudiants inscrits, commençant leur parcours académique en début 2011 et le finissant en 2014, c'est-à-dire quatre ans d'études. C'est ainsi que l'UFPA devient la deuxième université publique du Brésil à créer la licence en LS, après Santa Catarina, située au sud du Pays.

C'est aussi en 2010 que le Ministère de l'Education en partenariat avec l'UFPA octroie le projet «LIBRAS pour tous », à travers de l'appel d'offre 'PROEXT 2010/N 5', sous ma responsabilité, et dont le pôle universitaire bénéficiaire est le Campus de Soure. Ce projet avait comme but de sensibiliser la population des régions de l'Etat de Pará vis-à-vis de l'éducation des sourds à partir des pôles de l'UFPA éparpillés un peu partout dans l'intérieur de Pará. Nous avons assuré une formation de presque 300 heures de cours de LIBRAS et conférences multidisciplinaires autour de la surdité. Tout cela se faisait par voie visuelle. Le projet a permis à l'UFPA d'acquérir une grande quantité d'ouvrages sur le handicap de manière générale et notamment sur l'éducation des personnes sourdes et aveugles, ainsi que beaucoup d'équipements informatiques. Pendant toute l'année d'activités (2010/2011), le projet assurait des bourses de stage à un étudiant de chaque pôle de l'UFPA et nous avons pu créer à Soure le Laboratoire d'Education Inclusive, le premier de toute l'UFPA. L'un des objectifs de ce projet était de faire la collecte de LS dans ces régions où nous développions nos actions et cela a été fait dans quelques-unes de ces villes de l'intérieur de l'Etat. Seulement, la méthodologie appliquée ne correspond pas à un modèle linguistique cohérent compte tenu du manque de connaissance sur tout ce qui concerne une collecte de LS, dès sa conception méthodologique aux objectifs à atteindre lors du traitement du matériel collecté.

Il faut savoir que nous n'avions pas encore pris connaissance du modèle sémiologique de l'Université Paris 8. Donc, toutes les démarches concernant l'éducation des sourds suivaient la politique d'inclusion dont l'objectif premier était d'apprendre la LIBRAS aux sourds. L'équipe de professeurs attachés à l'enseignement de la LIBRAS et du Portugais L2, ainsi comme les autres professionnels impliqués dans 
ce processus de construction des stratégies éducatives pour les sourds, ne considéraient pas la LS pratiquée par les sourds, soit en famille soit entre eux, en tant qu'une langue à part entière et structurée. Nous considérions tous ces gestes du mime et essayions de montrer aux sourds les «bons signes » en LIBRAS. Voici notre point de départ et dont les principes nous ont guidés lors de nos premières collectes de LS des sourds de Soure, Ile de Marajó. Ainsi, comme nous le verrons un peu plus loin, mon parcours méthodologique consiste en deux démarches distinctes, réalisées dans des périodes bien précises, la première en 2008 et la deuxième en 2013, après avoir pris connaissance du modèle sémiologique de l'Université Paris 8.

Je décide donc de me rendre en France pour un séjour d'études dans le domaine de la linguistique de LS. Mon premier contact avec l'Université Paris 8 était avec professeur Ivani Fusellier en 2008 par le biais du directeur de 1'INJS, Monsieur JeanFrançois Dutheil. Ayant perdu contact avec Paris 8 le directeur me renoue avec cette université par le biais de Monique Gendrot, interprète de l'INJS, qui m'envoie à Brigitte Garcia, m'accueillant au sein du CNRS/Paris 8 en septembre 2012 pour un stage postdoctoral. Dans ce même mois, Ellen Formigosa, l'enseignante sourde de LIBRAS de la licence en LIBRAS et Portugais L2 de l'UFPA, s'amène en France où elle est inscrite en Master I à l'Université Paris 8.

Et c'est dans cette université, en contact avec les chercheurs de la linguistique de la LS, que je me suis rendue compte de l'importance de l'iconicité dans les LS pratiquées par les sourds de Soure, je comprends que ces LS ont une organisation propre, qu'il ne s'agit pas de mime, mais des langues à travers lesquelles les sourds sont capables de communiquer pleinement. A mon sens, ceci marque un moment historique dans ce parcours de collecte des LS des sourds en question, car c'est la première fois que j'appréhende leur langue en tant qu'un discours structuré et pas en tant que signe fragmenté en dehors du contexte. Et toute la richesse de cette interaction communicative s'ouvre à mes yeux. Je découvre à travers leur LS leur culture imprégnée dans chaque mouvement et expression du visage, je comprends finalement le rapport entre le sens et l'iconicité, je suis capable d'identifier les transfers et le rôle du regard d'après chaque contexte et les lier avec leur culture environnante, enfin, toute la théorie que je venais d'absorber d'une façon un peu abrupte s'ouvrait là, dans la pratique, et mes yeux étaient maintenant sensibles à cette perception grâce à cette plongée dans les écrits de Cuxac 
(1996), Fusellier-Sousa (2004), Garcia ; Fusellier; Sallandre (2009) et tant d'autres lecture auxquelles j'étais souvent renvoyée par le biais des séminaires au CNRS/Paris 8 .

En mars 2013, je suis partie pour la collecte des LS des sourds de Soure/Île de Marajó et dont le corpus devait non seulement servir à mes réflexions méthodologiques de collecte des LS en question mais aussi au mémoire de Master II d'Emmanuella Martinod, étudiante inscrite à Paris 8 sous la direction de Brigitte Garcia, intitulé «Les LS pratiquées par des sourds isolés de Marajó » et dont la soutenance a eu lieu en septembre 2013. Ce même corpus est utilisé par Ellen Formigosa, dont le mémoire porte sur la variation linguistique des LS et qui a été soutenu en 2014, sous la direction d'Ivani Fusellier.

Pour la première fois nous avons fait une collecte de ces LS d'après un regard scientifique et adéquat selon le modèle sémiologique de Paris 8. Cela représente un grand pas vers la mise en place d'un travail fondé sur des supports théoriques approfondis concernant les approches méthodologiques et linguistiques des LS pratiquées par les sourds éparpillés partout dans l'Etat de Pará. Tracer la mémoire de cette trajectoire, qui a commencé en 2006, nous semble être la première tâche à accomplir avant toute démarche.

Cet acheminement de faits et de recherche autour de la LS, en tant que postdoctorante attachée au CNRS/Paris 8, me permet d'affiner les conceptions méthodologiques du point de vue théorique et technologique concernant la collecte des LS faite en 2013. J'exposerai ce parcours méthodologique depuis 2008, de façon à montrer l'évolution de ces procédures très distinctes et dans les démarches et dans le temps.

\section{La collecte des LS en 2008}

Une première collecte des données en LS a été réalisée dans le but d'apprendre la LIBRAS et le portugais L2 aux sourds de Soure, sans se soucier de la façon dont ils communiquaient et moins encore de leur insertion dans la société.

La méthodologie qui était toute à fait intuitive consistait à montrer des images aux sourds d'après lesquelles ils devaient s'exprimer dans leur LS et ensuite l'interprète en LIBRAS qui était leur interlocuteur leur montrait le signe en LIBRAS. 
Dans un troisième moment les sourds visualisaient le mot en portugais qui correspondait à l'image en question.

Il n'y avait pas donc aucune préoccupation à préserver leur façon de communiquer, qui était propre à la manière dont les sourds de Soure percevaient le monde d'après leur culture et les implications de rapport d'identité avec la culture sourde.

$\mathrm{Du}$ point de vue sociologique la problématique de la barrière de communication ne se posait pas et du point de vue linguistique nous n'avions pas de support théorique à suivre car la démarche consistait à comprendre l'insertion des sourds en milieu scolaire par le biais de l'apprentissage de la langue portugaise écrite sans tenir compte de la diversité des LS pratiquées par les sourds de ce municipe.

\section{La collecte des LS en 2013}

Les procédures méthodologiques de la collecte des LS en 2013 ont été établies en cinq démarches : 1) nature des données ; 2) méthode de collecte ; 3) collecte des données : types de situations de communication envisagées ; 4) procédures de collecte ; et 5) spécification technique. L'entretien semi-directif a été organisé en dixsept questions, comme suit, en vue de construire la grille de métadonnées ${ }^{4}$ des sourds interviewés. J'expose ensuite les contraintes de terrain, qui ont provoqué des changements de procédure méthodologique, suivies des observations et analyses concernant chaque situation de communication réalisée pendant la collecte des LS des sourds en question. Pour conclure, je fais une mise au point concernant les résultats selon les divers aspects constatés tout au long de ce travail de terrain.

\subsection{Nature des données}

Corpus des LS de Soure/Ile de Marajó - Brésil: Narration, récits de vie, dialogue et description.

\subsection{Méthode de collecte}

Entretien semi-directif et récit libre «in situ » :

\footnotetext{
${ }^{4}$ Exemple : figure 1.
} 
- Entretien semi-directif « in situ »: l'enquêteur et le locuteur sourd sont accompagnés de l'interlocuteur privilégié entendant du locuteur. Cet interlocuteur qui fait office d'interprète :

1 Quel âge avez-vous?

2 Êtes-vous appareillé ? Avez-vous un implant cochléaire?

3 Parlez-moi de votre famille. Vos parents sont-ils sourds ou entendants ?

4 Combien de frères et sœurs avez-vous ? Sont-ils tous entendants ?

5 Quel âge ont-ils?

6 Avec le(s)quel(s) d'entre eux vous entendez-vous le mieux ?

7 Vivez-vous tous ensemble?

8 Lorsque vous êtes tous ensemble, comment faites-vous pour vous faire comprendre?

9 Avez-vous toujours vécu à Soure ?

10 Êtes-vous allé à l'école ? Si oui, laquelle ? A partir de quel âge y êtes-vous allé et quand avez-vous arrêté d'y aller?

11 Comment se passaient vos journées dans cette école ? Etiez-vous toujours avec les entendants? L'instituteur s'adressait-il à vous en portugais du Brésil, en langue des signes brésilienne (LIBRAS) ou s'aidait-il du LPC ?

12 Est-ce que vous avez un métier? Si oui, lequel ?

13 Comment faites-vous pour vous faire comprendre sur votre lieu de travail ?

14 Etes-vous croyant? Si oui, fréquentez-vous régulièrement dans un lieu de culte ?

15 L'office est-il compréhensible pour vous ? Est-il interprété en LIBRAS ?

16 Rencontrez-vous régulièrement d'autres sourds ? Si oui, où vous retrouvez-vous ? Avec quelle fréquence?

17 Quelle langue utilisez-vous pour communiquer ? Est-ce la même que celle que vous utilisez dans votre famille ? Est-ce la LIBRAS ?

- Récit libre « in situ»

Les contextes communicatifs de récit libre «in situ» on été réalisés selon la méthodologie utilisée par Ivani Fusellier, où l'interlocuteur privilégié du sourd sert d'intermédiaire dans l'intéraction proposée. Nous avons aussi exposé la mère sourde et son fils entendant dans un environnement organisé avec des jouets - mère et fils jouant ensemble - en sorte qu'ils communiquent sans aucun médiateur dans le but d'observer leur échange. Cette situation nous a permis de constater que cet échange se passe notamment par le regard et qu'elle communique avec son fils en montrant ce qu'elle veut dire par le pointage ou en lui montrant 
des objets. Dans un autre contexte, nous avons laissé deux sourds adultes communiquer dans une salle, sans médiateur, avec deux caméras dans des angles différents (frontal et latéral).

\subsection{Collecte des données : types de situations de communication envisagées}

Bipartite : le sourd et son interlocuteur privilégié entendant - sourds isolés

Echange mère sourde/enfant entendant

Sourds en contact avec la LIBRAS

Sourds dans les offices religieux

Interaction entre sourds

- Bipartie: la communication se fera entre le sourd et son interlocuteur entendant d'après un entretien semi-directif "in situ" : dialogue sur sa situation familiale et sociale, sa scolarité, son métier et sur le rapport de parenté avec son interlocuteur. Dans un deuxième moment, les sourds seraient demandés de raconter leur vie à partir de ce contexte initialement préparé à la conversation (récit de vie).

- Mère/enfant: dialogue libre entre la mère sourd et l'enfant entendant dans le contexte d'un "corpus écologique". La mère serait demandée de raconter son histoire de vie (récit de vie).

- Sourds/LIBRAS : l'échange doit se faire à partir des situations d'interaction entre les personnes qui maîtrisent la LIBRAS et qui sont en contact direct avec les sourds dans des démarches pédagogiques dont le but serait celui de les instruire (soit par rapport à l'enseignement du portugais soit dans d'autres contextes de transmission de connaissances dont la LIBRAS serait la langue utilisée). Les sourds seraient demandés de raconter des récits de vie concernant leurs métiers.

- Sourds/offices religieux : dans le cadre de la transmission religieuse, la collecte des données se ferait pendant un échange de communication entre celui qui communique avec les sourds en LS et ceux-ci.

- Sourds/sourds : dialogue « in situ » où les interlocuteurs auraient un profil diversifiés (isolés ou pas) et seraient censés interagir entre eux - « corpus écologique ». 


\subsection{Spécification technique}

Les plans seront choisis en fonctions des objectifs requis de prise de vue « comprenant le locuteur sourd en interaction avec un autre interlocuteur ». Les espaces seront sélectionnés selon l'accord des sourds et leurs lieux habituels seront privilégiés et dans des conditions d'éclairage adéquates. «L'organisation schématique des séances d'enregistrements » doit constituer un registre de procédure méthodologique et jointe au cheminement parcouru lors de la collecte des données, ainsi que la « biographie condensé de la chaque formateur » (FUSELLIER, 2004).

\subsection{Contraintes du terrain}

Compte tenu de la barrière de communication les sourds ne s'approprient pas des biens culturels et nous n'avons pas pu les exposer au film sur les légendes locales car ils ne les connaissaient pas ;

La collecte des données pendant l'office religieux n'a pas eu lieu dû à une organisation préalable exigée et nous n'avions pas assez de temps pour la réaliser ;

Concernant l'espace de prise d'images, nous avons privilégié les résidences des sourds car nous croyions qu'ils seraient plus à l'aise et seulement un sourd a communiqué de façon très spontanée et naturelle chez lui. L'une des sourdes a eu un blocage lors de l'échange et nous avons décidé de continuer le travail dans un endroit plus neutre, ce qui a fonctionné et les sourds se sont sentis plus à l'aise pour communiquer.

Ces contraintes m'ont obligée à organiser différemment les situations de communication comme suit ci-dessous :

- Deux groupes mixtes (garçon et fille) - espace neutre

- Une fille sourde et sa mère entendante - espace neutre

- Une fille sourde et sa mère entendante - chez elle

- Une mère sourde et son fils entendant - espace neutre

- Un sourd et sa sœur - chez lui

- Un sourd seul devant la caméra - chez lui

$\rightarrow$ Les groupes mixtes (2)

1. $\mathrm{V} / \mathrm{R}$ 
2. $\mathrm{J} / \mathrm{S}$

$\rightarrow \underline{\text { Une fille sourde et sa mère entendante }}$

3. $\mathrm{S} / \mathrm{M}$

$\rightarrow \underline{\text { Une fille sourde et sa mère entendante }}$

4. $\mathrm{Ma} / \mathrm{M}$

$\rightarrow \underline{\text { Une mère sourde et son fils entendant }}$

5. $\mathrm{M} / \mathrm{H}$

$\rightarrow \underline{\text { Un sourd et sa sœur }}$

6. $\mathrm{E} / \mathrm{S}$

$\rightarrow \underline{\text { Un sourd seul devant la caméra }}$

7. $\mathrm{E}$

\section{Conclusion}

Les résultats que nous avons obenus pendant la présente recherche sont fondés sur trois aspects essentiels pour notre approche méthodologique : Contexte situationnel des sourds d'après les données recueillies lors des outils méthodologiques appliqués en mars 2013 ; dimensions sociale et politique et le contexte linguistique.

Tout d'abord, les LS pratiquées par les sourds isolés en question ne sont pas considérées, par un grand nombre de chercheurs, en tant que langues en ayant une structure linguistique, étant tout simplement vues comme des gestes iconiques ne méritant pas d'être introduites dans les procédures pédagogiques concernant l'éducation des sourds. Seulement un sourd parmi les 6 interviewés sait lire et écrire et maîtrise la LIBRAS car il a suivi un parcours scolaire ailleurs, c'est également le seul sourd non isolé. Mais il comprend les LS pratiquées par les autres sourds et cela nous montre qu'il est possible d'envisager un enseignement de la LIBRAS à partir de leurs LS. Les autres 5 sourds ne sont pas scolarisés ou ont fréquenté l'école très peu. Ceux-ci restent chez eux et font des tâches domestiques car, selon leurs parents, l'école ne leur apprend rien. L'une des sourdes a un enfant et ils communiquent surtout par le regard. Ces sourds travaillent tous dans des métiers qu'ils ont appris en observant la pratique des autres. Comme ils ne peuvent pas communiquer en dehors de leurs familles à travers leurs LS, ils restent à l'écart des manifestations culturelles locales et ne connaissent pas la 
tradition du peuple qu'ils côtoient. Les tâches quotidiennes sont traduites dans leurs LS avec un grand rapport iconique avec la réalité.

Concernant leurs LS, la situation était assez complexe. Ils communiquaient au travers la LS qu'ils créent dont les gestes sont compréhensibles par leur entourage familial en sachant qu'ils ont souvent un membre de la famille qui est leur interlocuteur privilégié. Il s'agissait d'un groupe très hétérogène concernant la scolarité et la maîtrise de la LIBRAS en milieu scolaire car, à Soure, leur professeur ne communiquait pas en LIBRAS avec eux et très peu parmi eux poursuivait un parcours scolaire à long terme. L'apprentissage du portugais n'était pas envisagé d'après une méthodologie appropriée. Ainsi, nous avons constaté que les politiques publiques n'étaient pas appliquées d'après ce qui est prévu dans la loi de 2005 : interprètes, enseignement de la LIBRAS et du portugais L2. Cela signifie que les sourds en question vivent en condition d'isolement social dans ce sens où ils ne s'appropriaient pas de leurs biens culturels et ne sont pas ainsi en condition d'exercer leur citoyenneté de façon participative dans la société. Outre cela, nous avons pu vérifier que ces personnes sourdes se trouvent en situation de vulnérabilité car elles ne disposent pas des moyens pour acquérir leur autonomie dans l'environnement où elles habitaient. Et pour cela même, les sourds sont enfermés chez eux comme une mesure de sécurité qui, en réalité, leur prive de tout contacte normal avec les personnes qui les entourent. Le corpus recueilli sera aussi analysé selon le modèle sémiologique de l'iconicité, ce qui nous permettra d'observer les aspects pertinents concernant la structure de leurs LS.

Dans l'univers de la communauté sourde enquêtée nous avons des indicateurs révélateurs de dimensions sociale et politique impliquées dans la présente recherche, tels que:

- Nombre de sourds non scolarisé ;

- Nombre de sourds qui ne communiquent pas entre eux soit en leur LS soit en LIBRAS;

- Nombre de sourds isolés ;

- Nombre de sourds ayant parents entendants (qui pensent que la seule façon d'insérer les sourds dans la société est de leur apprendre à parler) ;

- Nombre de professeurs non qualifiés dans le domaine de l'éducation des sourds; 
- Nombre de dispositifs issus des politiques publiques qui ne sont pas appliquées dans le réseau scolaire de l'enseignement public;

Même si le but de ce travail n'est pas celui de quantifier ces indicateurs, ils nous montrent tout autant que nous sommes face à une réalité complexe concernant l'insertion des sourds dans la société locale. Et si nous partons de l'hypothèse que La situation d'isolement des sourds de Soure - Ile de Marajó est due en grande partie à la non reconnaissance de leur LS en milieu scolaire de façon à favoriser un enseignement à partir de leur référentiel de culture en LS, dont la gestuelle est marquée par l'iconicité révélatrice de cette culture, cela dévoile les dimensions sociale et politique a prendre en compte dans les contexte de notre recherche, à savoir : la sociale, où l'insertion sociale des sourds isolés doit passer obligatoirement par la reconnaissance de leurs LS en milieu scolaire et à partir desquelles un nouveau modèle d'enseignement de la LIBRAS doit être proposé ; et la dimension politique qui consiste à appliquer les politiques publiques pour l'insertion des sourds isolés dans la société en leur permettant l'accès aux biens culturels et aux mêmes opportunités assurées aux entendants.

Finalement, le contexte linguistique nous a permit de souligner quelques aspects pertinents concernant les LS pratiquées par les sourds enquêtés :

- prédominance de pointage et TTF dans les échanges entre eux ;

- rapport entre le mouvement labial et la gestualité (ex : café/geste de boire en tenant une tasse ; pâte/mouvement cassant les pâtes), dans leur échange avec les entendants - dans ce type de de transfer l'objet est remplacé par l'action et son référentiel iconique ;

- les LS pratiquées entre eux sont restées en sa majorité leur façon de communiquer malgré le contact qu'ils ont eu avec la LIBRAS pour une période très courte. Par exemple, le fils entendant $(\mathrm{H})$ dont la mère $(\mathrm{Ma})$ est sourde garde sa propre façon de communiquer malgré le fait que sa mère ait été exposée à la LIBRAS, ce qui porte d'ailleurs une certaine confusion de communication lorsqu'elle fait un signe en LIBRAS et son fils réponds dans la LS qu'ils avaient l'habitude de parler. 
Dans l'image 1, elle lui demande de nommer l'objet qu'elle lui montre [LAPIN] en lui faisait un signe en LIBRAS, les deux mains en configuration 37 articulant contre les latérales de la tête et en mouvement dirigé vers le devant qui marquent les oreilles comme le trait saillant de l'animal, sauf que ce signe veut dire [CHEVAL], elle confond les signes pour [LAPIN] et [CHEVAL] que l'on lui avait appris et vite elle corrige et fait le signe correct en LIBRAS - la configuration en 37, même point d'articulation et en mouvement vers le derrière), or, son fils ne tient rien en compte et lui répond dans sa LS : la configuration en 48, point d'articulation devant la bouche et en mouvement vertical de haut en bas de la pointe des doigts.

Figure 1 - La mère sourde demande son enfant de nommer l'objet qu'elle lui montre (le lapin)

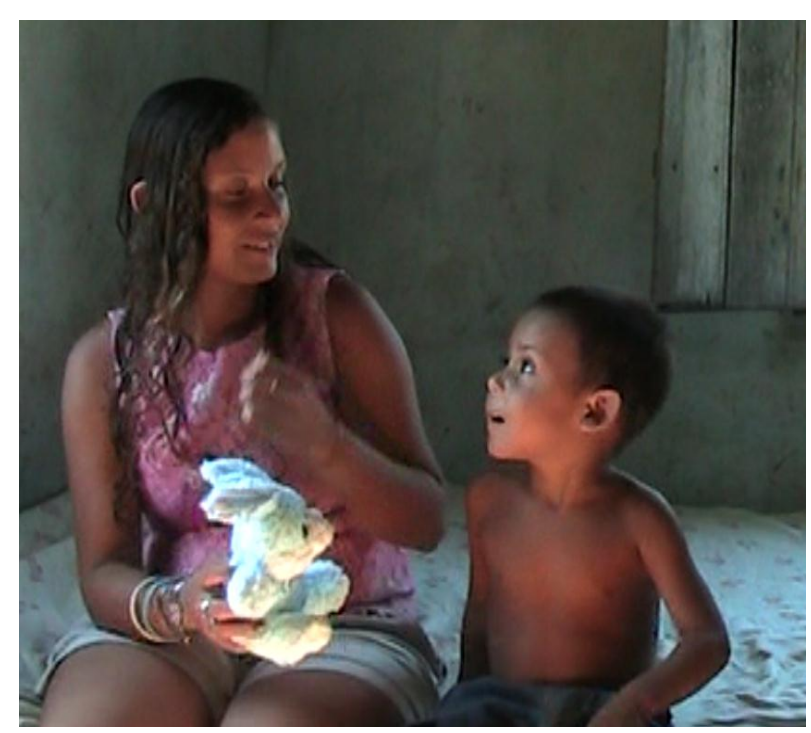

Source: CARLIEZ, M.L.S., 2013.

Ensuite, la mère refait [LAPIN] - image 2, en configuration 33 et mouvement vers le devant, point d'articulation sur la latérale de la tête. L'on remarque ainsi qu'elle a été exposée à la LIBRAS mais la langue n'est pas encore systématisée car elle confond les configurations et le mouvement des mains. 
Figure 2 - LS de Soure [LAPIN]

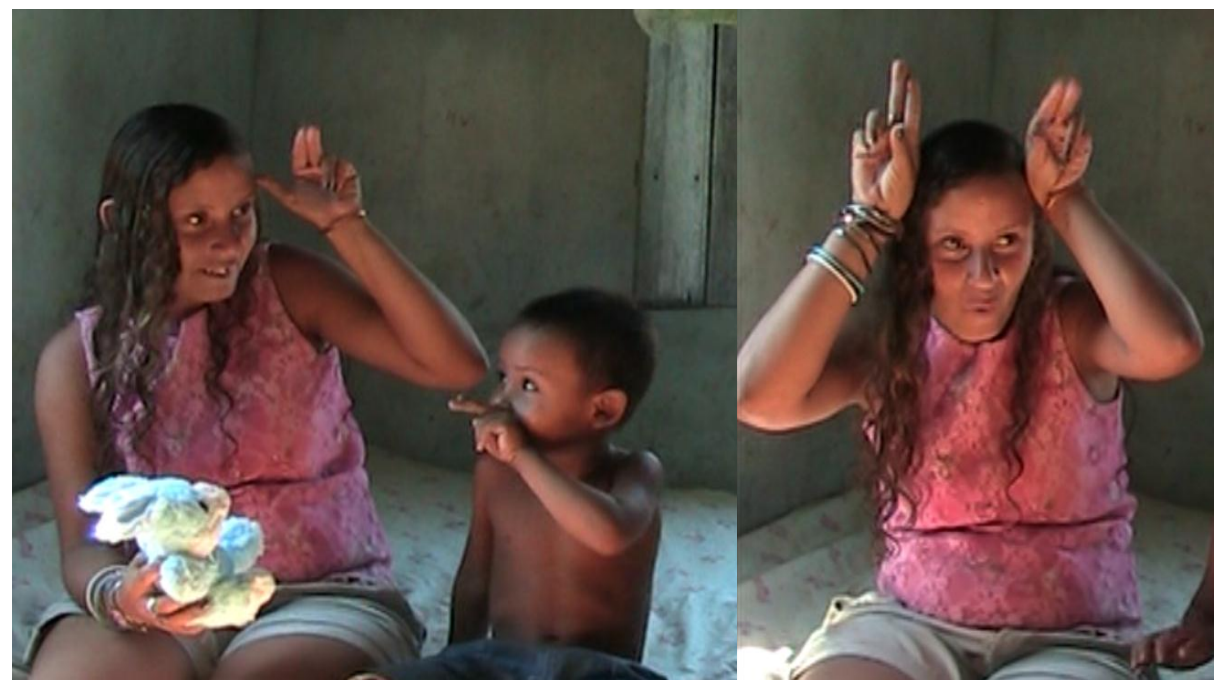

Source: Ibidem, 2013.

Figure 3 - LS de Soure [POISSON]

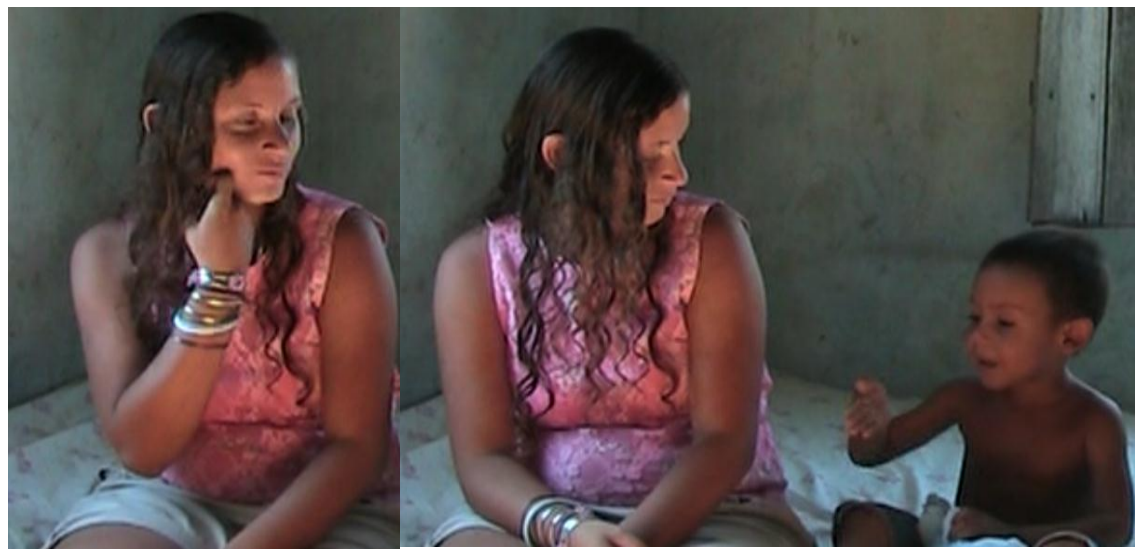

Source: Ibidem, 2013.

L'image 3 nous montre une situation similaire où la grand-mère lui dit de demander à sa mère comment dit-on [POISSON] et il fait la configuration en 56 en mouvement zigzag qui évoque le poisson nageant et la mère lui fait le signe en LIBRAS - configuration en 12 et point d'articulation sur la latérale du menton ; il ne retient pas l'information et continue à parler dans la LS qu'il communiquait avec sa mère.

Dans l'une des situations de conversations mixtes $(\mathrm{J} / \mathrm{S})$, le sourd $(\mathrm{J})$ et la sourde (S) parlent du bruit que fait la corde accrochée à la poulie lorsque l'on fait monter du fond du puis le seau rempli d'eau. (S) explique que le bruit est perçant et cela en frottant la pointe de l'ongle du doigt indicateur droit contre la paume de sa main gauche en faisant un mouvement de va-et-vient sur la surface. Ces sourds qui ne s'étaient jamais 
parlé ont les mêmes signes iconiques pour [POULIE] et dans leur interaction gestuelle ils produisent ce signe au même instant pour dire que le bruit vient du contact de la corde avec la poulie, comme si dans un registre implicite de compréhension simultanée un même mot était prononcé en même temps par les interlocuteurs. Ceci constate la fluidité du discours gestuel et soulève d'emblée une question d'ordre socio-culturel lié au phénomène de création en LS par ces sourds en question. Il s'agirait, d'après nous, d'une cristallisation des SGI dans le discours des sourds de ces micro-communautés, où nous apercevons deux mécanismes de stabilisation des structures gestuelles : soit le processus de contamination langagière qui ne pourrait être envisagé que par les interlocuteurs entendants soit l'interférence du référentiel de culture ancré dans leur environnement d'entourage qui ne prendrait pas en compte la contamination gestuelle des entendants. Nous croyons que c'est le cas ici de la [POULIE] qui serait une création gestuelle des sourds de Soure sans aucune contamination gestuelle des entendants surtout parce que la préoccupation d'exprimer [POULIE] gestuellement est inhérente à l'effet qu'elle cause aux oreilles des sourds, ce qui ne serait pas aperçu comme une gêne particulière chez les entendants. Ce phénomène socio-culturel lié au processus de création des LS chez les sourds vivant dans ces micro-communautés est révélatrice de l'autonomie langagière des sourds, même en situation d'isolement. Donc, ce fait nous montre encore une fois que, comme les langues orales, les langues visuelles-gestuelles se structurent autour d'une pragmatique liée aux éléments symboliques de la culture où vivent ces personnes. Après nous avons bien entendu pour les LS de ces microcommunautés, et d'ailleurs pour toutes les langues, l'émergence de l'économie gestuelle qui serait peut-être à l'origine d'un certain éloignement iconique de départ. Ainsi, la contamination par la LIBRAS ne serait pas, d'après notre perception, un facteur d'évolution de ces LS vers des formes stables, au contraire, la LIBRAS dans ce contexte se superpose aux LS pratiquées par ces sourds qui ont du mal à la systématiser dans leur contexte discursif. Nous observons ainsi que cet éloignement des SGI ne s'opère même pas dans certains contextes présents ici et ces SGI restent d'ailleurs des formes stables dans les LS pratiquées par les sourds de ces micro-communautés, dont quelques aspects ont d'ailleurs attiré notre attention, par exemple, lors de notre contact avec les LS des sourds de Fortalezinha qui ont subi, aussi à leur tour, une contamination de la LIBRAS. 


\section{Références}

CUXAC, C. Fonctions et structures de l'iconicité des langues des signes. thèse de doctorat d'Etat, Université René Descartes, Paris V, 1996.

FUSELLIER-SOUZA, I. Sémiogenèse des langues des signes: Étude de langues des signes primaires (LSP) pratiquées par des sourds brésiliens. Thèse de doctorat en Sciences du Langage, Université Paris 8, 2004.

GARCIA, B. ; SALLANDRE, M-A. ; FUSELLIER-SOUZA, I. Rôle du pointage dans l'expression de la définitude en langue des signes. Colloque international du geste au signe, le pointage dans les langues orales et signées, organisé par l'UMR 8163 "Savoirs, Textes, Langage" (CNRS et universités Lille 3 \& Lille 1), 4 et 5 juin, Université de Lille 3, Villeneuve d'Ascq. (conférence non publiée), 2009.

GENDROT, M. L'interprète en institution: à la croisée des regards. Mémoire de DFSSU d'Interprétation LSF/Français, soutenu à l'Université Vincennes Saint Denis Paris 8, 2004.

Avenant au mémoire du DFSSU (2004). Mémoire soutenu à l’Université Vincennes Saint Denis - Paris 8, 2008.

GRINEVALD, C.; BERT, M. Linguistique de terrain sur langues en danger. OPHRYS, Paris, 2010.

MARIE-ROSE, E.F.F. Étude de la variation linguistique de la LS au brésil dans l'enseignement de la Libras. Dissertação de Mestrado defendido na Université Vincennes Saint Denis - Paris 8, 2014.

MARTINOD, E. Les LS pratiquées par des sourds isolés de Marajó. Dissertação de Mestrado defendida na Université Vincennes Saint Denis- Paris 8, 2013. 